\title{
Demanda residencial de energia elétrica em Minas Gerais: 1970-2002
}

\section{Palauras-chave}

demanda residencial, energia elétrica, co-integração, Minas Gerais, VECM.

Classificação JEL C32, D12, R21.

\section{Key words \\ residential demand, electricity, co-integration, VECM.}

JEL Classification C32, D12, $\mathrm{R} 21$.

\section{Resumo}

Este trabalho teve como objetivo obter uma estimativa para a demanda residencial de energia elétrica em Minas Gerais, no período de 1970 a 2002. Especificamente, pretendeu-se estimar a elasticidade-preço e a elasticidaderenda dessa demanda. Após identificar que as séries estudadas eram não-estacionárias, optou-se pela utilização do conceito de cointegração, sendo estimado um Modelo de Correção de Erros Vetoriais (VEC Model). Os resultados obtidos mostraram que a demanda estudada é mais sensível a variações na renda que no preço da energia elétrica, embora tanto a elasticidade-renda quanto a elasticidade-preço se mostraram inferiores à unidade. Conclui-se também que esta última elasticidade é maior em Minas Gerais que no Brasil, sugerindo padrões diferenciados de consumo dessa energia entre os diversos Estados do País.
Leonardo Bornacki de Mattos Mestrando em Economia Aplicada pela Universidade Federal de Viçosa

João Eustáquio de Lima Professor do Departamento de Economia Rural da Universidade Federal de Viçosa

\section{Abstract}

The objective of this study was to obtain an estimate for residential electricity demand in Minas Gerais in the period from 1970 to 2002. Specifically, the goal was to estimate price and income elasticities. After determining that the series under study were non-stationary, we chose to use the Co-integration approach, estimating a Vector Error Correction Model (VEC Model). The results obtained show that the demand studied is more sensible to variations in the income than to the price of the electricity, although both elasticities are inferior to the unit. It was also concluded that price-elasticity is bigher in Minas Gerais than it is for Brazil as a whole, suggesting differentiated usage of this energy among the different states in the Country. 


\section{1_Introdução}

O setor elétrico brasileiro, refletindo uma tendência observada em diversos outros países, vem sofrendo uma série de mudanças na sua condição de funcionamento. Tais mudanças começaram a ser implementadas a partir de 1993, através da Lei n. 8.631/93 e, posteriormente, com a Lei n. 8.987/95 e Lei n. 9.074/95 em 1995.

De acordo com Pires et al. (2001), a Lei n. 9.074/95 constituiu os pilares do funcionamento do novo modelo e do início do processo de privatização de ativos públicos, iniciado pela venda das distribuidoras federais e, posteriormente, acompanhado pela venda das empresas estaduais.

Pires (1999) cita ainda que duas outras leis foram de grande importância para o novo modelo do setor. A Lei n. 9.427/96, que, por ter criado a Agência Nacional de Energia Elétrica (ANEEL), representou um marco na reforma regulatória do setor elétrico brasileiro, e a Lei n. 9.648/98, que, entre outras coisas, definiu as regras de entrada de novas empresas, as tarifas e a estrutura de mercado. Essa lei ainda criou o Mercado Atacadista de Energia (MAE), que foi importante medida na direção de estimular a competição nos segmentos de geração e comercialização da energia elétrica (Ministério de Minas e Energia - MME, 2003a).
Entretanto, os objetivos principais dessas mudanças não foram alcançados na íntegra, mantendo comprometida a expansão do sistema e, ao gerar uma nova crise financeira no setor, reintroduziu a questão da inadimplência nos fluxos financeiros intra-setoriais, influenciando negativamente a geração de recursos das empresas para a manutenção adequada da prestação dos serviços (MME, 2003a).

Visando suprir as deficiências do modelo então em vigor, foi publicado, em dezembro de 2003, um novo Modelo Institucional para o setor (MME, 2003b). O novo projeto regulatório pretende, entre outros pontos, criar um ambiente propício à retomada de investimentos, de forma a garantir o fornecimento de energia elétrica em quantidade e qualidade requeridas pelo mercado consumidor.

Nesse modelo, o estudo da demanda de energia elétrica tem importância estrutural e conjuntural, tanto no planejamento quanto no gerenciamento do setor elétrico brasileiro, por exercer impacto direto e/ou indireto sobre as seguintes decisões: programas decenais de expansão da geração, transmissão e distribuição; planejamento da operação dos sistemas; programas de investimento das empresas; contratos de compra e venda de energia entre empresas; preço da ener- 
gia no mercado atacadista; programa de licitação de obras do agente regulador, entre outros (Eletrobrás, 2003).

Há ainda que se considerar o fato de que, com o novo modelo institucional, caberá às distribuidoras de energia elétrica a contratação do total do mercado por elas previsto, sendo essas os principais agentes responsáveis pelas estimativas de demanda. De modo geral, o mercado de uma distribuidora se limita a parcelas de mercado de um único Estado, indicando a importância de análises de demanda regionalizadas.

Sendo assim, este trabalho pretendeu contribuir com uma estimativa da demanda de energia elétrica para Minas Gerais, mais precisamente com a demanda da classe Residencial. Especificamente, procurou determinar a elasticidadepreço e a elasticidade-renda dessa demanda. Este Estado é o segundo maior consumidor de energia elétrica, tanto na região Sudeste quanto no mercado nacional. Em 2002, foi responsável pelo consumo de $23,2 \%$ de toda a energia elétrica consumida na região Sudeste e por $12,7 \%$ do consumo desse tipo de energia no Brasil (Eletrobrás, 2002). A classe Residencial, por sua vez, é a segunda maior consumidora do Estado, representando aproximada- mente $18 \%$ do consumo de energia elétrica (Fundação João Pinheiro, 2002).

\section{2_Consumo Residencial de energia elétrica em Minas Gerais}

A energia elétrica, embora amplamente utilizada nas residências, não se constitui no principal energético consumido no setor Residencial do Estado de Minas Gerais. No período de 1983 a 2002, exceto nos anos de 1998 a 2000, ocupou o posto de terceira fonte mais consumida, sendo superada pela lenha e pelo Gás Liquefeito de Petróleo (GLP). Entretanto, entre todas as fontes de energia, a elétrica foi a que mais aumentou sua participação no consumo dessa classe. Em média, representou $13,7 \%$ do total, atingindo valor máximo de 20,6\% em 1999 (Cemig, 2003).

Segundo Andrade e Lobão (1997), essa ampliação no consumo de energia elétrica nas residências é decorrente da entrada de aparelhos eletroeletrônicos no ambiente doméstico, o que aumenta o seu estoque, bem como do maior uso do equipamento já existente. No período de 1970 a 2002, o consumo da classe Residencial mineira cresceu à taxa média de 8,3\% a. a. Entre as classes consumidoras, foi a que apresentou a maior taxa de cres- 
cimento. Os únicos anos em que foi presenciada queda na quantidade consumida de energia elétrica foram 2001 e 2002, como resultado do programa de racionamento do consumo, implantado em junho de 2001. O Gráfico 1 apresenta a evolução do consumo Residencial de energia elétrica em Minas Gerais, no período 1970-2002.
Ao longo dessas três décadas, o comportamento do consumo de energia elétrica pela classe Residencial foi influenciado pela dinâmica das tarifas de fornecimento, assim como pela renda dos consumidores. A Tabela 1 apresenta as taxas geométricas de crescimento dessas variáveis, inclusive do consumo, em cada uma das décadas.

\section{Gráfico 1 Evolução do consumo de energia elétrica pela classe} Residencial em Minas Gerais, 1970-2002

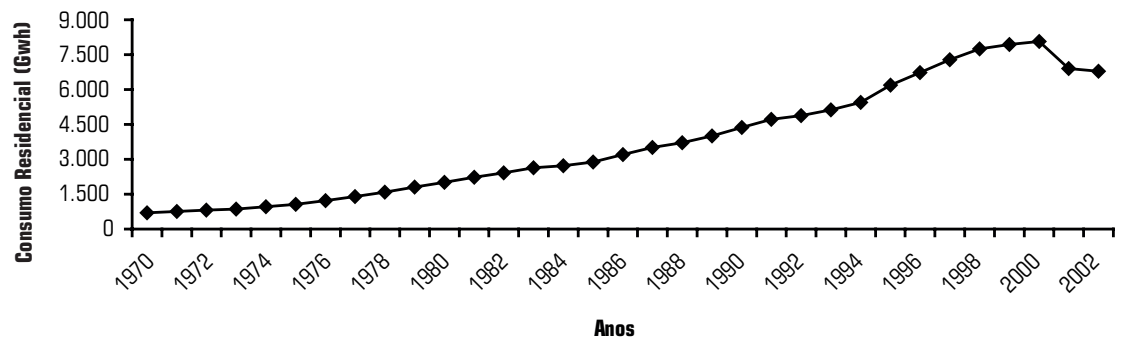

Fonte: Fundação João Pinheiro (2004).

Tabela 1_Taxas geométricas de crescimento do PIB per capita' das Tarifas Médias Residenciais e do consumo Residencial em Minas Gerais ( $\%$ ao ano)

\begin{tabular}{|c|c|c|c|c|}
\hline & $1970-1979$ & 1980-1989 & 1990-1999 & 1970-2002 \\
\hline PIB per capita & $9,8^{*}$ & $1,1^{* *}$ & $2,0^{*}$ & $2,2^{*}$ \\
\hline Tarifas & $-3,3 *$ & $-7,8^{*}$ & $1,1^{\mathrm{NS}}$ & $-3,3 *$ \\
\hline Consumo Residencial & $11,2^{*}$ & $7,8^{*}$ & $7,4^{*}$ & $8,3^{*}$ \\
\hline
\end{tabular}

$\left(^{*}\right)$ valores significativos a $1 \%$;

(**) valores significativos a $5 \%$;

NS indica valores não significativos.

Fonte: Dados da pesquisa.

\author{
Para a análise proposta \\ por este trabalho, \\ considerou-se o PIB real per \\ capita do Estado de \\ Minas Gerais como proxy \\ para a renda média familiar
} deste Estado. 
Durante a década de 70, o consumo residencial de energia elétrica foi impulsionado principalmente pelo crescimento expressivo da renda do Estado, mas também sofreu a influência das reduções tarifárias. Nesse mesmo período, o crescimento do número de residências eletrificadas foi pouco inferior ao do consumo, resultando em pequeno incremento no consumo médio por residência.

Nos anos 80, década que ficou conhecida como a "década perdida", houve forte retração na atividade econômica do País, o que não foi diferente em Minas Gerais. Diante de uma renda per capita praticamente estagnada, o principal fator que influenciou positivamente o consumo de energia foi a drástica redução das tarifas médias de fornecimento. Segundo Branco (1996), o governo federal utilizava desse expediente como medida de controle do processo inflacionário. Novamente se observou, em Minas Gerais, incremento no consumo médio das residências.

Foi na década de 90 que a classe Residencial menos elevou seu consumo, apesar de o PIB per capita ter apresentado crescimento pouco superior à década anterior. Ao contrário do ocorrido nos anos 80 , as tarifas (residenciais) de energia elétrica não decresceram, sendo mantidas praticamente constantes ao longo dos dez anos. O número de consumidores cresceu em média 4,9\% a.a. e, inferior ao consumo total, resultou na elevação do consumo médio por residência, que alcançou 1.831,5 KWh/ano em 1999.

Outro fator que favoreceu a expansão do consumo residencial de energia elétrica, nessas três décadas, foi o comportamento dos preços reais dos eletrodomésticos. Houve queda acentuada no preço médio real desses aparelhos o que, segundo Silva (2001), permitiu que as famílias aumentassem o estoque domiciliar desses bens, contribuindo, dessa forma, para o aumento do consumo de energia elétrica nas residências.

Embora a classe Residencial tivesse elevado continuamente seu consumo de energia elétrica ao longo do período 1970/2002, sua participação no consumo total dessa energia em Minas Gerais registrou dois comportamentos distintos: durante a década de 70, a classe Residencial reduziu sua parcela no consumo total do Estado, quando caiu de 15,9\%, em 1970, para $11,5 \%$ em 1979. A partir de 1980, ano em que esse índice atingiu seu valor mínimo, $11,2 \%$, iniciou-se uma trajetória de crescimento, que foi mantida até o ano de 1999, quando atingiu participação máxima, respondendo por 19,6\% de toda a energia elétrica consumida no 
Estado. Após apresentar ligeira redução em 2000, a classe Residencial voltou a ter sua participação reduzida nos anos seguintes, alcançando 17,6\% em 2002.

\section{3_Demanda Residencial de energia elétrica}

A Teoria do Consumidor, ao abordar a demanda por bens e/ou serviços, baseiase na maximização da utilidade do consumidor, sujeita a sua restrição orçamentária. Sendo assim, a demanda de um bem $i$ pode ser vista como resultado do seguinte problema microeconômico:

Maximizar $U\left(X_{i}\right)$

Sujeito a: $P_{i} X_{i}+P_{n} X_{n} \leq R$

em que: $U\left(X_{i}\right)$ é a função de utilidade do consumidor;

$P_{i}$ é o preço do bem $i$;

$P_{n}$ é o preço de todos os $n$ bens consumidos;

$X$, é a quantidade consumida do bem $i$;

$X_{n}$ é a quantidade consumida dos $n$ bens;

$\mathrm{R}$ é a renda do consumidor;

(2) é a restrição orçamentária.

A solução do problema anterior é dada por:
$X_{i}=f\left(P_{i}, P_{n}, R\right)$

em que a quantidade demandada do bem $i$ é função das variáveis $P_{i}, P_{n}$ e $\mathrm{R}$, definidas anteriormente.

Entretanto, existem alguns bens que apresentam algumas particularidades na relação entre preço e quantidade demandada. Esse é o caso da água e da energia elétrica, que, por serem ofertados e demandados segundo tarifas que variam por blocos de consumo, não têm apenas um preço de equilíbrio, mas uma lista de preços, dificultando a especificação de suas demandas.

Tal especificidade faz com que um dos pontos mais discutidos na literatura econômica, que aborda a demanda de energia elétrica, seja a especificação da variável preço. Há autores que defendem a utilização do preço marginal, enquanto outros, o uso de preços médios. O preço marginal é o preço cobrado por unidade de consumo referente ao bloco onde recai a quantidade total consumida, enquanto o preço médio é obtido pela divisão do valor total pago pela quantidade total consumida (Silva, 2001).

Taylor (1975), após realizar uma revisão sobre 11 estudos de demanda de energia elétrica, concluiu que a especificação mais correta de uma função de demanda de um bem que tem preço em 
"

2 Alguns desses estudos são: Modiano (1984), Andrade e Lobão (1997), Braga (2001), Silva (2001), Schmidt e Lima (2004). bloco é aquela em que há tanto o preço médio quanto o preço marginal. Bjorner et al. (2001) defendem que, em geral, a utilização de preços marginais deve ser preferida ao uso de preços médios, uma vez que, sendo o preço médio função da quantidade consumida, este se torna uma variável endógena em vez de exógena.

Os estudos que têm sido realizados para o Brasil utilizam a tarifa média nas estimativas da função de demanda. ${ }^{2}$ Os autores acreditam que essa variável é uma boa escolha para responder a alterações na quantidade demandada de energia elétrica. Andrade e Lobão (1997) lembram ainda que informações referentes às tarifas marginais não são disponíveis, ratificando o uso da tarifa média.

Em concordância com esses autores, neste estudo foi utilizada a tarifa média de energia na estimação da demanda. Acredita-se também que essa é a variável de conhecimento do consumidor quando esse define ou altera seu padrão de consumo de energia elétrica.

De forma geral, a demanda de energia elétrica é abordada como função de fatores econômicos e do estoque dos equipamentos que necessitam de energia elétrica para seu funcionamento, podendo ser representada da seguinte forma:

$Q=f\left(T_{t}, R_{t}, E E_{t}\right)$ em que: $Q_{t}$ é a quantidade demandada de energia;

$T_{t}$ é a tarifa de energia;

$\mathrm{R}_{t}$ é a renda do consumidor; $E E_{t}$ é o estoque de equipamentos que necessitam de energia elétrica para seu funcionamento; té o tempo.

De acordo com a teoria, aumentos (reduções) na tarifa de energia, coeteris paribus, provocam redução (aumento) na quantidade consumida, indicando relação inversa entre as duas variáveis. Tais alterações fazem com que os consumidores procedam de duas formas: alterando a utilização dos equipamentos já existentes, ou adquirindo novos e mais eficientes equipamentos. Assim, a demanda é um resultado indireto do uso de equipamentos que necessitam de energia elétrica para seu funcionamento (Berndt, 1991; Kamerschen e Porter, 2004).

A renda dos consumidores influencia a demanda de energia elétrica positivamente, de forma direta e indireta. Quando há aumento nessa variável, mantendo-se as demais variáveis constantes, ocorre mudança na restrição orçamentária à qual o consumidor está sujeito, permitindo-lhe maior consumo dos bens, entre os quais, está a energia elétrica. Esse primeiro efeito depende do estoque 
de equipamentos já existente, sendo alterada apenas sua taxa de utilização. O consumidor pode também decidir pela aquisição de novos equipamentos, aumentando o estoque e, conseqüentemente, o consumo de energia elétrica.

$\mathrm{O}$ estoque de equipamentos, assim como a renda do consumidor, influencia positivamente a demanda de energia elétrica. No curto prazo, esse estoque é considerado fixo, e a demanda restrita a alterações na sua taxa de utilização. No longo prazo, o estoque é flexível, podendo variar de acordo com alterações na renda, no preço dos equipamentos e em outros fatores. De forma geral, pode ser indicado por:

$E E_{t}=f\left(R_{t}, \ldots, R_{t-n}, P E_{t}, \ldots P E_{t-n}\right)$

em que: $E E_{t}$ é o estoque de equipamentos;

$\mathrm{R}_{t}$ é a renda do consumidor; $P E_{t}$ é o preço dos equipamentos; té o tempo;

$n$ é o número de períodos passados.

Como pode ser observado a partir de (5), o estoque de equipamentos possui relação contemporânea com as variáveis que o determinam, mas também é influenciado pelos valores passados dessas variáveis.
Substituindo-se (5) em (4), obtémse uma expressão para a demanda de energia elétrica, sendo apresentadas as relações diretas e indiretas dessa demanda com as variáveis que a influenciam.

$Q_{t}=f\left[T_{t}, R_{t}, E E_{t}\left(R_{t}, \ldots, R_{t-n}, P E_{t}, \ldots, P E_{t-n}\right)\right]$

Entretanto, uma medida para o estoque dos equipamentos tem sido considerada problemática em razão da qualidade dos dados. Segundo Holtedahl e Joutz (2004), alguns procedimentos foram desenvolvidos, a fim de evitar a utilização dessa variável, mas os resultados obtidos normalmente estão abaixo do esperado.

Sendo assim, neste estudo optouse por não considerar explicitamente o estoque de equipamentos na especificação da demanda de energia elétrica, acreditando-se que os efeitos dessa variável sobre a demanda de energia elétrica são captados pelas variações nas tarifas, na renda dos consumidores e no preço dos eletrodomésticos.

\section{4_Metodologia}

\subsection{Modelo econométrico}

A função de demanda de energia elétrica estimada neste trabalho pode ser representada por uma função do tipo Cobb- 
A funco de demanda do

3 A função de demanda do tipo Cobb-Douglas também foi utilizada por Modiano (1984), Andrade e Lobão (1997), Silva (2001), Holtedahl e Joutz (2004) e Schmidt e Lima (2004).

4 Sobre esse assunto, Nordin (1976) já havia constatado que, tanto nos modelos que utilizaram o preço em bloco como naqueles que usaram o preço médio, há o problema de simultaneidade.
Douglas. Essa forma funcional tem sido muito utilizada em economia, principalmente em estudos de demanda. Uma das suas principais propriedades é que as elasticidades são constantes e iguais aos coeficientes das variáveis. Tal função pode ser representada da seguinte forma: ${ }^{3}$

$Q_{t}=k R_{t}^{\beta_{1}} T_{t}^{\beta_{2}} E L_{t}^{\beta_{3}} e^{v_{t}}$

Tomando-se o logaritmo natural (ln) em ambos os lados de (7), a equação pode ser apresentada em uma forma linear:

$Q_{t}=\alpha+\beta_{1} R_{t}+\beta_{2} T_{t}+\beta_{3} E L_{t}+v_{t}$

em que: $\alpha=\ln k$ é o intercepto da função de demanda;

$Q_{t}$ é o logaritmo natural da quantidade consumida de energia elétrica pela classe Residencial no tempo $t$;

$\mathrm{R}_{t}$ é o logaritmo natural da renda da classe Residencial no tempo $t$; $T_{t}$ é o logaritmo natural da tarifa residencial de energia elétrica no tempo $t$;

$E L_{+}$é o logaritmo natural do preço dos eletrodomésticos no tempo $t$;

$v_{t}$ é o termo de erro com pressuposições usuais; té o tempo, medido em anos.

Das equações (7) e (8) esperou-se que o consumo de energia elétrica res- pondesse positivamente a aumentos na renda dos consumidores $\left(\beta_{1}>0\right)$, e negativamente a aumentos da tarifa da energia elétrica e do preço dos eletrodomésti$\cos \left(\beta_{2}\right.$ e $\left.\beta_{3}<0\right)$. Os parâmetros $\beta_{1}, \beta_{2}$ e $\beta_{3}$ podem ser interpretados como as elasticidades da demanda de energia elétrica em relação à renda, ao preço da energia elétrica e ao preço dos eletrodomésticos, respectivamente.

\subsection{Método de estimação}

A utilização da tarifa média de energia como proxy para o preço da energia elétrica traz algumas implicações sobre as estimativas das funções de demanda. Uma vez que há dependência recíproca entre a tarifa média e a quantidade consumida de energia elétrica, têm-se duas variáveis determinadas endogenamente no modelo, sendo (que) uma delas (é) um regressor. Assim, a provável simultaneidade entre as variáveis pode violar a hipótese de ausência de correlação entre o termo de erro e o regressor, o que inviabilizaria o uso do Método dos Mínimos Quadrados Ordinários (MQO). ${ }^{4}$

Há, segundo Schmidt e Lima (2004), duas metodologias que podem ser utilizadas nessas circunstâncias: Modelos de Equações Simultâneas e/ou Técnicas de Co-Integração. 
Neste trabalho, optou-se por utilizar a segunda opção, mais precisamente mediante os procedimentos de estimação e os testes desenvolvidos por Johansen $(1988,1991)$ e ainda Johansen e Juselius (1990). Tal metodologia consiste em estimar, por Máxima Verossimilhança, o(s) vetor(es) de co-integração, valendo-se da modelagem de um Vetor Auto-Regressivo (VAR). Numa segunda etapa, com base no vetor de co-integração obtido, estima-se um Mecanismo de Correção de Erros (MCE), com vetor de co-integração. Cabe ressaltar que esses modelos têm sido freqüentemente utilizados nos estudos mais recentes de demanda de energia elétrica. Por exemplo: Andrade e Lobão (1997), Silk e Joutz (1997), Silva (2001), Holtedahl e Joutz (2004), Narayan e Smyth (2003) e Schmidt e Lima (2004).

A opção por esses modelos pode ser também justificada pelas propriedades estatísticas das séries temporais das variáveis analisadas, que, geralmente, são séries não-estacionárias. ${ }^{5}$ Durante muito tempo, a recomendação usual para se trabalhar com séries não-estacionárias era que essas deveriam ser diferenciadas. Isso porque muitas séries econômicas são integradas de ordem 1 [I(1)], ou seja, têm uma raiz unitária e, portanto, tornam-se estacionárias na primeira diferença (Coelho, 2002).
Entretanto, segundo Enders (1995), esse procedimento restringe a análise a um contexto de curto prazo e, conforme ressaltado por Gujarati (2000), a maior parte da teoria econômica é enunciada como uma relação de longo prazo entre as variáveis na forma de nível, e não na forma de primeira diferença. Para a análise de longo prazo entre variáveis não-estacionárias, o procedimento mais adequado, quando aplicável, é o de co-integração.

O conceito de co-integração foi introduzido por Engle e Granger (1987) e tem sido amplamente empregado na análise de séries temporais. Diferentemente da recomendação padrão de que as séries não-estacionárias devem ser utilizadas em primeira diferença, a co-integração, quando aplicável, permite que regressões que envolvem esse tipo de variável sejam realizadas sobre seus níveis, sem que se incorra no problema da regressão espúria, além de não se perder informação de longo prazo, o que ocorre quando são utilizadas séries diferenciadas.

Engle e Granger (1987) procuraram mostrar que, apesar de duas (ou mais) variáveis serem não-estacionárias, é possível haver uma (ou mais) combinação linear entre elas que seja estacionária. Esse conceito, segundo Enders (1995), pode ser definido da seguinte forma:
5 Segundo Gujarati (2000), uma série é (fracamente) estacionária se suas média e variância forem constantes ao longo do tempo e o valor da covariância entre dois períodos de tempo depender apenas da distância ou defasagem entre os dois períodos, e não do período de tempo efetivo em que a variância é calculada. 
6 Uma série é integrada de ordem $d\left(y_{t} \sim I(d)\right)$ se ela precisa ser diferenciada $d$ vezes para se tornar estacionária $\left(\Delta^{d} y_{t}\right.$ é estacionária). Assim, uma série estacionária é uma série $\mathrm{I}(0)$.

7 Contudo, para os trabalhos de natureza empírica, interessa apenas o caso particular em que $d=b=1$, tal que $\beta X_{t} \sim I(0)$.

8 O agrupamento desses vetores em uma matriz dá origem a uma matriz $\beta$, de dimensão $r \times n$, denominada matriz de co-integração (Verbeek, 2000).
Os componentes do vetor $X_{t}=\left(X_{1 t}, X_{2 t}, \ldots, X_{n t}\right)$ são ditos co-integrados de ordem $\mathrm{d}, \mathrm{b}$, indicado por $X_{t} \sim C I(d, b)$ se:

1. todos componentes de $X_{t}$ são inte$\operatorname{grados}^{6}$ de ordem $d\left(X_{t} \sim I(d)\right)$;

2. existe um vetor $\beta=\left(\beta_{1}, \beta_{2}, \ldots, \beta_{n}\right)$ tal que a combinação linear $\beta X_{t}=\left(\beta_{1} X_{1 t}+\beta_{2} X_{2 t}+\ldots+\beta_{n} X_{n t}\right)$ é integrada de ordem $(d-b)$, sendo $b>0$. O vetor $\beta$ é chamado de vetor de co-integração. ${ }^{7}$

O sistema de variáveis econômicas encontrar-se-á em equilíbrio de longo prazo quando $\beta_{1} X_{1 t}+\beta_{2} X_{2 t}+\ldots+\beta_{n} X_{n t}=0$. De modo geral, se $X_{t}$ possui $n$ variáveis, é possível determinar $r \leq n-1$ vetores de co-integração. ${ }^{8} \mathrm{O}$ número de vetores de co-integração $(r)$ é igual ao número de vetores linearmente independentes e é conhecido como rank de co-integração. Entretanto, para qualquer escala $\lambda \neq 0$, a multiplicação $\lambda \times \beta$ dará origem a um "novo" vetor. De maneira similar, segundo Enders (1995), é comum utilizar uma das variáveis $\left(X_{i}\right)$ para normalizar o vetor $\beta$, fazendo seu coeficiente $\left.\beta_{i}\right)$ igual a 1. Para tanto, é necessário apenas definir $\lambda=1 / \beta_{i}$.

\section{3_ Fonte de dados \\ e operacionalização do modelo}

Como proxy para o preço da energia elétrica, foi utilizada a Tarifa Média Resi- dencial, em R \$/MWh, a preços constantes de 2003, fornecida pela Cemig. Como medida da renda dessa classe, foi utilizada a série do PIB per capita de Minas Gerais, em R\$/habitante, cuja fonte é a Fundação João Pinheiro (FJP), deflacionada pelo Deflator Implícito do PIB $(2003=100)$. Os dados do Consumo Residencial de energia elétrica, em MWh, também tiveram como fonte a FJP. Também foi utilizado o índice real de preços de eletrodoméstico, cuja fonte é a Fundação Getúlio Vargas, tendo como base o ano de $2003(2003=100)$. Todos os dados são anuais e referentes ao período de 1970 a 2002.

Para a operacionalização do modelo foi utilizado o software econométrico Eviews4.

\section{Resultados e discussões}

\section{1_ Teste de Raiz Unitária}

A utilização do conceito de co-integração só se justifica se as séries analisadas são não-estacionárias ou, em outras palavras, têm raiz unitária. Portanto, o primeiro procedimento foi a realização dos Testes de Raiz Unitária de Dickey-Fuller (DF) e de Dickey-Fuller Aumentado (ADF). Os resultados obtidos são apresentados na Tabela 2 . 
Tabela 2_ Testes de Raiz Unitária DF e ADF, realizados para as séries $Q_{t}, R_{t}, T_{t}$ e $E L_{t}$, para o período de 1970 a 2002

\begin{tabular}{|c|c|c|c|c|c|}
\hline \multirow{2}{*}{ Série } & \multirow{2}{*}{ Equação de teste" } & \multirow{2}{*}{$\begin{array}{l}\text { Número de } \\
\text { defasagens" }\end{array}$} & \multirow{2}{*}{$\begin{array}{c}\text { Estatística } \\
\text { de teste } \\
\text { (ADF) }\end{array}$} & \multicolumn{2}{|c|}{ Valor Crítico } \\
\hline & & & & $5 \%$ & $1 \%$ \\
\hline$Q_{t}$ & constante & 1 & -2.293 & -2.960 & -3.661 \\
\hline$\Delta Q_{t}$ & constante e tendência & 0 & -3.559 & -3.215 & -4.284 \\
\hline $\mathrm{R}_{t}$ & constante & 4 & -2.910 & -2.971 & -3.689 \\
\hline$\Delta R_{t}$ & sem constante e sem tendência & 3 & -3.148 & -1.953 & -2.650 \\
\hline$T_{t}$ & constante & 0 & -1.420 & -2.957 & -3.653 \\
\hline$\Delta T_{t}$ & sem constante e sem tendência & 0 & -5.844 & -1.952 & -2.641 \\
\hline$E L_{t}$ & constante e tendência & 0 & -1.523 & -3.557 & -4.273 \\
\hline$\Delta E L_{t}$ & constante e tendência & 0 & -5.124 & -2.960 & -3.661 \\
\hline
\end{tabular}

$\left.{ }^{*}\right)$ somente foram incluídas nas equações de teste as constantes e tendências estatisticamente significativas; ${ }^{9}$

(**) foi utilizado o número de defasagens que minimizou o Critério de Informação de Schwarz.

Fonte: Dados da pesquisa.

Os resultados apresentados na Tabela 2 indicam que, para as séries em nível, não se pode rejeitar a presença de raiz unitária aos níveis de significância de $1 \mathrm{e}$ $5 \%$. Portanto, todas as séries possuem raiz unitária e são não-estacionárias. $\mathrm{O}$ teste realizado para as séries em primeira diferença indicou que, ao nível de significância de $5 \%$, se pode rejeitar a presença de raiz unitária. Assim, todas as séries em diferença têm a mesma ordem de integração, ou seja, são todas I(1), e é possível que sejam co-integradas.

\section{2_ Teste de co-integração e relação de longo prazo entre as variáveis}

Atendido o pré-requisito de que as séries sejam integradas de mesma ordem, o passo seguinte foi testar a existência de cointegração entre elas, utilizando o procedimento de Johansen, tomando-se por base a especificação de um modelo VAR.

Assim como no teste de raiz unitária, para a realização do teste de co-integração, é necessário à correta determinação do número de defasagens e da pre-
Os valores críticos para o teste de significância da constante e da tendência podem ser obtidos em Enders (1995). 


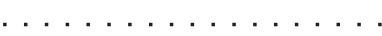

10 Segundo Moreira et al.

(1996), o modelo a ser

estimado deve

necessariamente ser

parcimonioso quanto ao

número de variáveis, sob pena

de perda excessiva de graus de

liberdade na estimação dos

seus parâmetros.

11 Foram utilizados os procedimentos descritos em Enders (1995).

12 Entre 6/2001 e 2/2002, o programa foi implantado nas regiões Sudeste, Centro-Oeste e Nordeste. Na região Norte, teve menor duração, estando presente entre 8/2001 e 1/2002.

13 Algumas dessas mudanças são: maior conscientização dos consumidores quanto ao uso desse tipo de energia, utilização de lâmpadas fluorescentes em substituição às incandescentes, substituição de equipamentos elétricos por equipamentos que utilizam outro tipo de energia, etc. sença ou não de termos determinísticos a ser incluídos. Os termos determinísticos podem ser uma constante, uma tendência ou ainda uma variável Dummy.

Com a finalidade de determinar o número de defasagens $(p)$ do modelo VAR, foram adotados dois critérios: o primeiro, a decisão pelo número de defasagens $(p)$ que minimizou os Critérios de Informação de Schwarz e de Hannan-Quinn. O segundo, o teste de significância da defasagem mais alta. Os resultados estão apresentados na Tabela 3 .

Com base na Tabela 3 nota-se que, de acordo com o Critério de HannanQuinn, o modelo VAR deve conter duas defasagens. Entretanto, o Critério de Schwarz e o teste de significância estatística da mais alta defasagem indicam que o modelo VAR deve conter apenas uma defasagem. Uma vez que os modelos VAR têm como uma de suas características principais o consumo de muitos graus de liber- dade, optou-se por incluir apenas uma defasagem em cada uma de suas variáveis. ${ }^{10}$

Definida a ordem do VAR, o passo seguinte foi determinar a inclusão ou não de termos determinísticos, como constante e tendência. ${ }^{11}$ A conclusão foi a de que o modelo deveria conter tanto uma constante quanto uma tendência.

Como variável exógena do modelo, foi incluída uma Dummy, indicada por $D$, com o objetivo de captar uma possível mudança estrutural da demanda de energia elétrica em função do programa de racionamento do consumo. ${ }^{12} \mathrm{~A}$ variável $D$ assumiu o valor 1 para os anos de 2001 e 2002, e o valor 0 (zero) para os demais anos. Considerando-se a possibilidade de que algumas mudanças ocorridas durante o racionamento, e que tenham reduzido o consumo de energia elétrica, ainda permaneçam em vigor, espera-se que o coeficiente dessa variável apresente sinal negativo. ${ }^{13}$

Tabela 3_ Definição do número de defasagens do modelo VAR

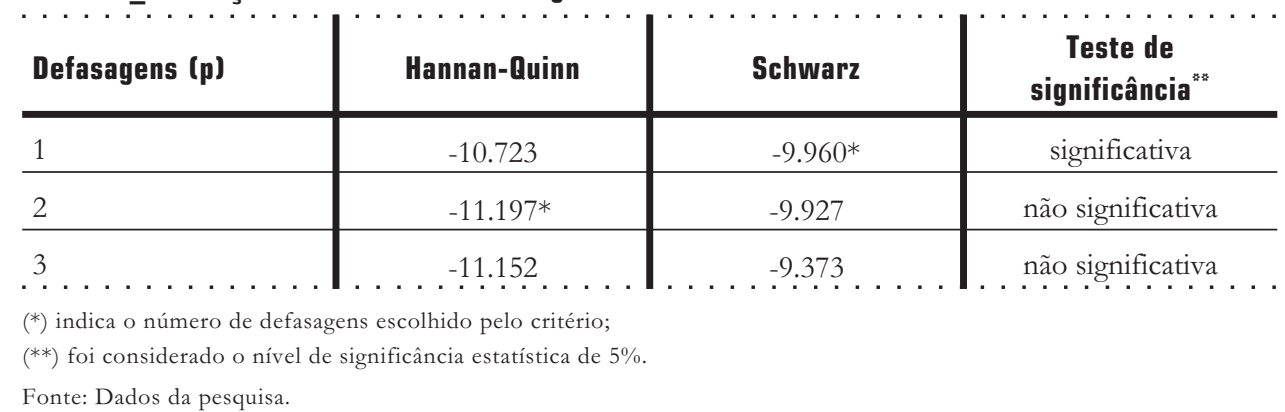


Determinada a correta especificação do modelo VAR, realizou-se o teste proposto por Johansen (1988), com o objetivo de encontrar, caso exista relação de longo prazo entre as variáveis, o(s) vetor(es) de co-integração. Para tanto, baseou-se nos dois testes desenvolvidos por Johansen e Juselius (1990), capazes de determinar o número de vetores de cointegração. São eles: o teste do traço, comumente indicado por $\lambda_{\text {trace }}$ e o teste do máximo autovalor (eigenvalue), indicado por $\lambda_{\max }$. As Tabelas 4 e 5 apresentam os resultados dos testes do traço e do máximo autovalor, respectivamente.

Tabela 4_ Teste do traço para co-integração, realizado para as séries $Q_{t}, R_{t}, T_{t}$ e $E L_{t}$, para o período de 1970 a 2002

\begin{tabular}{c|c|c|c|c}
$\begin{array}{c}\text { Hipótese } \\
\text { nula }\left(\mathrm{H}_{\mathbf{0}}\right)\end{array}$ & $\begin{array}{c}\text { Hipótese } \\
\text { alternativa }\left(\mathrm{H}_{\mathbf{1}}\right)\end{array}$ & $\begin{array}{c}\text { Estatística do } \\
\text { teste }\end{array}$ & \multicolumn{2}{|c}{ Valor crítico } \\
\cline { 3 - 5 } & $\mathrm{r}>0$ & 106,3036 & 62,99 & $\mathbf{5 \%}$ \\
\hline $\mathrm{r}=0$ & $\mathrm{r}>1$ & 41,4720 & 42,44 & 40,05 \\
\hline $\mathrm{r} \leq 1$ & $\mathrm{r}>2$ & 19,4502 & 25,32 & 30,45 \\
\hline $\mathrm{r} \leq 2$ & $\mathrm{r}>3$ & $7,0311 \ldots$ & $12,25 \ldots$ & $16,26 \ldots$ \\
$\ldots$
\end{tabular}

Fonte: Dados da pesquisa.

Tabela 5_Teste do máximo autovalor para co-integração, realizado para as séries $Q_{t}, R_{t}, T_{t}$ e $E L_{t}$, para o período de 1970 a 2002

\begin{tabular}{|c|c|c|c|c|}
\hline \multirow{2}{*}{$\begin{array}{l}\text { Hipótese } \\
\text { nula }\left(\mathrm{H}_{0}\right)\end{array}$} & \multirow{2}{*}{$\begin{array}{c}\text { Hipótese } \\
\text { alternativa }\left(H_{1}\right)\end{array}$} & \multirow{2}{*}{$\begin{array}{c}\text { Estatística do } \\
\text { teste }\end{array}$} & \multicolumn{2}{|c|}{ Valor crítico } \\
\hline & & & $5 \%$ & $1 \%$ \\
\hline $\mathrm{r}=0$ & $r=1$ & 64,8315 & 31,46 & 36,65 \\
\hline$r=1$ & $r=2$ & 22,0217 & 25,54 & 30,34 \\
\hline$r=2$ & $r=3$ & 12,4190 & 18,96 & 23,65 \\
\hline$r=3$ & $r=4$ & 7,0311 & 12,25 & 16,26 \\
\hline
\end{tabular}

Fonte: Dados da pesquisa. 
Segundo o teste do traço, a hipótese nula de que o posto da matriz de co-integração é nulo $(r=0)$ é rejeitada, tanto a $1 \%$ quanto a $5 \%$ de significância. Portanto, há no mínimo 1 vetor de co-integração que estabelece as relações de equilíbrio de longo prazo entre as variáveis. A análise da Tabela 4 indica que a hipótese nula de que há no máximo 1 vetor de co-integração $(r \leq 1)$ não pode ser rejeitada aos níveis de $1 \%$ e $5 \%$. Portanto, o teste do traço indica que, para esses níveis de significância, existe 1 vetor de co-integração.

De acordo com o teste do máximo autovalor, a hipótese nula de que o posto da matriz de co-integração é nulo $(r=0)$, assim como no teste do traço, é rejeitada, tanto a $1 \%$ quanto a $5 \%$ de significância. Portanto, o teste indica que há no mínimo 1 vetor de co-integração entre as variáveis. A hipótese nula de que o posto da matriz é igual a 1 $(r=1)$, ou seja, há 1 vetor de co-integração, não pode ser rejeitada em nenhum dos níveis de significância estatística apresentados na Tabela 5 .

Portanto, os dois testes utilizados indicaram que existe 1 vetor de co-integração. Assim, pode-se dizer que as variáveis são co-integradas, havendo uma relação de equilíbrio de longo prazo entre elas. O vetor de co-integração obtido, normalizado para a variável $Q_{t}$, é apresentado na Tabela 6.

Tomando-se por base o vetor de co-integração estimado, pode-se escrever a relação de equilíbrio de longo prazo do consumo de energia elétrica e seus determinantes e interpretar cada um dos parâmetros $\beta_{i}$ como a elasticidade da demanda de energia elétrica em relação à cada variável $X_{i}$. A equação (9) apresenta essa relação.

Tabela 6_Vetor de co-integração normalizado para a variável

\begin{tabular}{|c|c|c|c|c|c|}
\hline$Q_{t}$ & $\mathrm{~K}$ & $\mathrm{R}_{\mathrm{t}}$ & $T_{t}$ & $\mathrm{EL}_{\mathrm{t}}$ & Tendência \\
\hline 1,000000 & $-16,9948$ & $\begin{array}{c}-0,532420 \\
(0,04234)\end{array}$ & $\begin{array}{l}0,257866 \\
(0,04487)\end{array}$ & $\begin{array}{l}0,145934 \\
(0,04195)\end{array}$ & $\begin{array}{c}-0,042965 \\
(0,00614)\end{array}$ \\
\hline
\end{tabular}

(*) A estatística entre parênteses refere-se ao desvio padrão do parâmetro estimado.

Fonte: Dados da pesquisa. 
$Q_{t}=16,9948+0,532 R_{t}-0,258 T_{t}-0,146 E L_{t}+0,043 t$

Com base nesses resultados, pode-se afirmar que os sinais de todos os parâmetros da equação (9) estão de acordo com o sugerido pela teoria econômica. Conclui-se também que a demanda de energia elétrica, mesmo no longo prazo, é inelástica tanto em relação à renda dos consumidores quanto ao preço da energia (tarifas) e ao preço dos eletrodomésticos.

A elasticidade-renda estimada indica que, mantidas as demais variáveis constantes, o aumento de $1 \%$ na renda dos consumidores induz uma elevação de $0,532 \%$ na quantidade demandada de energia pela classe Residencial em Minas Gerais. Quando comparado a outros estudos, esse valor se mostrou intermediário aos 0,2132 e 0,9374 encontrados por Andrade e Lobão (1997) e Silva (2001), respectivamente. Entretanto, revelou-se praticamente igual ao resultado de 0,539 obtido por Schmidt e Lima (2004). Vale ressaltar que esse três trabalhos, utili- zados para comparação dos resultados, analisaram a demanda da classe Residencial do Brasil.

A demanda de energia elétrica se apresentou mais sensível às variações no preço da energia que às variações no preço dos eletrodomésticos, o que parece bastante razoável. Mantidas as demais variáveis inalteradas, o aumento de 1\% no valor das tarifas de energia provoca redução de $0,258 \%$ na quantidade demandada de energia. $\mathrm{O}$ valor de 0,258 se manifestou superior aos obtidos nos trabalhos citados anteriormente, o que indica que a demanda de energia elétrica da classe Residencial em Minas Gerais é mais sensível às variações das tarifas que a demanda dessa classe no Brasil.

\section{3_ Modelo de correção \\ de erros com vetor de co-integração}

Os procedimentos realizados até o presente momento foram úteis para determinar a relação de equilíbrio de longo prazo entre as variáveis. Entretanto, Engle e Granger (1987) demonstraram que, mesmo havendo relação de equilíbrio de longo prazo entre variáveis não-estacionárias (em nível), é possível que ocorra algum desequilíbrio no curto prazo. Nesse caso, a dinâmica de curto prazo é influenciada pela magnitude do desvio em relação ao equilíbrio de longo prazo, e deve haver algum mecanismo que conduza as variáveis para o equilíbrio. Esse mecanismo é conhecido como Mecanismo de Correção de Erros (MCE) e, pelo termo de erro (erro de equilíbrio), liga o comportamento de curto prazo das variáveis ao seu comportamento de longo 
prazo. Além disso, por meio do MCE, é possível determinar a velocidade com que os desequilíbrios são eliminados.

Sendo assim, foi estimado o MCE, valendo-se do modelo VAR utilizado para o teste de co-integração. A relação de longo prazo entre as variáveis, dada pelo vetor de co-integração e apresentada em (9), foi utilizada como variável explicativa do termo de correção de erros. Os modelos VAR, que têm um MCE com vetor de co-integração, são conhecidos na literatura como modelos VEC (Vector Error Correction).

Conforme ressaltado por Andrade e Lobão (1997), no caso específico de um modelo VAR com apenas uma defasagem das variáveis explicativas, o que acontece neste trabalho, a representação VEC fica determinada apenas com os termos de correção de erros. Dessa forma, tem-se:

$\begin{array}{ll}\text { Representação VAR(1) } & X_{t}=A_{0}+A_{1} X_{t-1}+\varepsilon_{t} \\ \text { Representação VEC } & \Delta X_{t}=A_{0}+\alpha_{1} u_{t-1}+\varepsilon_{t}\end{array}$

em que: $X_{t}=\left(Q_{t}, R_{t}, T_{t}, E L_{t}\right)$ é o vetor de variáveis dependentes e supostamente endógenas do modelo;

$A_{0}$ é o vetor de constantes; $A_{1}$ é a matriz de coeficientes das variáveis explicativas defasadas; $\varepsilon_{t}$ é o vetor de termos de erros supostamente normais, homocedásticos e independentes;

$\Delta X_{t}$ é o vetor de primeiras diferenças das variáveis de $X_{t}$;

$u_{t-1}$ é a relação de co-integração em $t-1\left(u_{t-1}=Q_{t-1}-16,9948-\right.$ $-0,532 R_{t-1}+0,258 T_{t-1}+$ $\left.+0,146 \mathrm{EL}_{t-1}-0,043 t\right)$ que é igual a $\beta^{\prime} X_{t-1}$, sendo $\beta$ o vetor de co-integração;

$\alpha$ é o vetor de coeficientes de correção de $u_{t-1}$ ou, em outras palavras, dos coeficientes de ajustamento.

Uma vez que o objetivo deste trabalho foi determinar as relações da demanda de energia elétrica com seus principais determinantes, optou-se por apresentar apenas a equação referente à variável $Q_{t}$. A Tabela 7 apresenta os resultados obtidos.

De acordo com os resultados apresentados na Tabela 7, conclui-se que todos os três coeficientes estimados são significativos ao nível de $1 \%$ de significância estatística. Portanto, a equação do modelo VEC, referente à variável $Q_{t}$, pode ser escrita conforme a equação (12).

$\Delta Q_{t}=0,083-0,434 u_{t-1}-0,193 D+\varepsilon_{t}(12)$ 
Tabela 7 _ Estimação do Modelo VEC referente à variável $Q_{t}$

\begin{tabular}{|c|c|c|}
\hline Variável Explicativa & Coeficiente & Estatística "t" \\
\hline$u_{t-1}$ & $-0,434$ & $-7,595$ \\
\hline Constante & 0,083 & 20,362 \\
\hline Dummy & $-0,193$. & $-11,644$ \\
\hline
\end{tabular}

Testes e Qualidade do Ajuste

\begin{tabular}{|c|c|}
\hline$R^{2}$ & 0,850 \\
\hline Estatística F & 82,279 \\
\hline Autocorrelação (LM-teste) & $23,063(0,112)$ \\
\hline Normalidade (Jarque-Bera) & $8,906(0,350)$ \\
\hline Heterocedasticidade (White) & $21,876(0,858)$ \\
\hline
\end{tabular}

Fonte: Dados da pesquisa.

Nota: As estatísticas entre parênteses referem-se ao p-valor.

O coeficiente de ajustamento estimado indica qual a proporção do desequilíbrio de curto prazo da demanda de energia elétrica é corrigida no período seguinte. Especificamente, o valor de 0,434 estabelece que, aproximadamente, 43,4\% da discrepância entre o valor efetivo e o valor de longo prazo, ou de equilíbrio, é corrigida a cada ano. Assim, a classe Residencial levaria entre dois e três anos para ajustar a quantidade demandada dessa energia a eventuais choques nas tarifas e/ou na renda dos consumidores.

A variável Dummy se mostrou significativa, e o seu sinal negativo indica que, nos anos de 2001 e 2002, o padrão de consumo de energia elétrica foi alterado para níveis inferiores aos observados antes do programa de racionamento do consumo. A inclusão dessa variável ocorreu muito mais pela preocupação em especificar corretamente o modelo VAR, que pelo interesse de obter estimativa para o parâmetro dessa variável. Portanto, julga-se de pequena relevância a interpretação da magnitude dessa mudança, uma vez que se trata de uma mudança transitória, que, dentro de pouco tempo, já terá sido eliminada.

O coeficiente de determinação encontrado foi de 0,850 , indicando que, aproximadamente, $85 \%$ das variações ocorridas na quantidade demandada de energia elétrica são explicadas pelo modelo ajustado.

$\mathrm{O}$ teste $\mathrm{F}$ mostrou-se altamente significativo, indicando que as variáveis expli- 
cativas são, conjuntamente, significativas para explicar o comportamento da quantidade demandada de energia elétrica.

Com base no teste do Multiplicador de Lagrange (LM-teste), a hipótese nula de que não há autocorrelação serial nos resíduos estimados não pode ser rejeitada aos níveis de significância estatística de 1,5 e $10 \%$. O teste de normalidade dos resíduos (Jarque-Bera) indicou que a hipótese nula de que os resíduos são normalmente distribuídos não pode ser rejeitada a esses mesmos níveis de significância. O teste de White mostrou que a hipótese nula de homocedasticidade nos resíduos também não pode ser rejeitada aos níveis de significância estatística considerados. Portanto, conclui-se que os resíduos estimados são não-autocorrelacionados, normalmente distribuídos e homocedásticos.

\section{6_Conclusão}

Este trabalho procurou apresentar uma estimativa da demanda de energia elétrica da classe Residencial em Minas Gerais, contribuindo para o preenchimento de uma das lacunas existentes nos estudos dessa demanda, qual seja, a da escassez de análises regionalizadas, uma vez que, em sua quase totalidade, as estimativas existentes abordam a classe Residencial do Brasil.

Os resultados mostraram que a demanda estudada é inelástica ao preço (tarifas), de forma que um ajuste na relação oferta/demanda, via preços, não parece ser a melhor alternativa, embora não se possa desconsiderar o relativo sucesso do programa de racionamento, implementado a partir de junho de 2001. Naquela ocasião, o não-cumprimento do limite de consumo por cada residência implicava a cobrança de multas e a possibilidade de corte do fornecimento de energia, esse que parece ter sido o principal responsável pelo sucesso de tal programa.

O fato de a demanda ter se mostrado mais sensível (menos inelástica) às variações das tarifas no Estado de Minas Gerais que no Brasil sugere que, em outros Estados brasileiros, essa elasticidade é ainda menor que a média nacional. Talvez, nesses Estados, o estoque de eletrodomésticos ainda não seja significativo, e a energia elétrica utilizada apenas em situações de primeira necessidade, como iluminação, banho e refrigeração de alimentos, sugerindo a existência de um mercado potencial.

Pode-se também imaginar, por essa constatação, políticas tarifárias dife- 
renciadas entre os Estados, condizentes com os respectivos padrões de consumo dessa energia. Para tanto, fazem-se necessárias estimativas das demandas de cada um dos Estados brasileiros, o que fica como sugestão para trabalhos posteriores. Sugere-se ainda a obtenção de estimativas das demandas das classes $\mathrm{Co}$ mercial e Industrial de Minas Gerais, o que permitiria políticas tarifárias diferenciadas também entre as classes, se evidenciadas diferenças significativas entre as elasticidades. 


\section{Referências bibliográficas}

ANDRADE, T. A; LOBÃO, W. J. A. Elasticidade renda e preço da demanda residencial de energia elétrica no Brasil. Rio de Janeiro: IPEA, 1997. (Texto para Discussão, n. 489).

BERNDT, E. R. The practice of Econometrics: classic and contemporary. [S. I.] AddisonWesley Publishing Company, 1991. Cap. 7, p. 306-337. 702 p.

BJORNER, T. B.; TOGEBY, M.; JENSEN, H. H. Industrial companies' demand for electricity: evidence from a micropanel. Energy Economics, v. 23, p. 595-617, 2001.

BRAGA, J. M. A modelagem da demanda residencial de energia elétrica. Rio de Janeiro, 2001. Dissertação (Mestrado em Economia) Instituto de Economia, Universidade Federal do Rio de Janeiro, Rio de Janeiro, 2001.

BRANCO, E. C. A reestruturação e a modernização do setor elétrico brasileiro. Belo Horizonte: Nova Economia, v. 6, n. 1, p. 105-141, 1996.

COElHO, A. B. A cultura do algodão e a questão da integração entre precos internos e externos. São Paulo, 2002. 136 p. Dissertação (Mestrado em Economia) - FEA, Universidade de São Paulo, São Paulo, 2002.
COMPANHIA ENERGÉTICA DE MINAS GERAIS - CEMIG. $18^{\circ}$ Balanço Energético do Estado de Minas Gerais - BEEMG. Belo Horizonte, 2003. Disponível em: <http://www.cemig.gov.br>. Acesso em: 22 jun. 2004

ELETROBRÁS. Departamento de Estudos Energéticos e Mercado (DEM). Boletim Anual. Sistema de Informações Estatísticas do Setor de Energia Elétrica (SIESE), 2002.

Disponível em:

<http://www.eletrobras.gov.br>. Acesso em: 3 mar. 2004.

ELETROBRÁS. Departamento de Estudos Energéticos e Mercado (DEM). Plano Decenal de Expansão 2003/2012. jan. 2003. Disponível em:

<http://www.eletrobras.gov.br>. Acesso em: 30 jan. 2004.

ENDERS, W. Applied Econometric Time Series. Nova York: John Wiley \& Sons, Inc., 1995. 433 p.

ENGLE, R. F.; GRANGER, C. W. Co-integration and error-correction: Representation, Estimation and Testing. Econometrica, v. 55, p. 251-76, 1987.

\section{FUNDAÇÃO JOÃO}

PINHEIRO. Anuário Estatístico de Minas Gerais - 2000/2001. Belo Horizonte, v. 9, p. 1-582, 2002.

\section{FUNDAÇ̃̃O JOÃO}

PINHEIRO. Consumo de energia elétrica em Minas Gerais, por classe de consumo: 1970/2002. Belo Horizonte, 2004. (Disponibilidade interna).

GUJARATI, D. N. Econometria Básica. 3. ed. São Paulo: Makron Books, 2000. 846 p.

HOLTEDAHL, P.; JOUTZ, F. L. Residential electricity demand in Taiwan. Energy Economics, v. 26, n. 2, p. 201-224, 2004.

\section{JOHANSEN, S. Statistical} analysis of cointegrating vectors. Journal of Economics Dynamics and Control, v. 12, p. 231-254, 1988.

JOHANSEN, S. Estimation and hypothesis testing of cointegrating vectors in Gaussian vector autoregressive models. Econometrica, v. 59, n. 6 p. 1551-1580, 1991.

JOHANSEN, S.; JUSELIUS, K. Maximum likelihood estimation and inference on cointegration with application to the demand for money. Oxford Bulletin of Economics and Statistics, v. 52, p. 169-209, 1990
KAMERSCHEN, D. R.; PORTER, D. V. The demand for residential, industrial and total electricity, 1973 - 1998. Energy Economics, v. 26, p. 87-100, 2004.

MINISTÉRIO DE MINAS E ENERGIA. Proposta de Modelo Institucional do Setor Elétrico. jul. 2003a. Disponível em: <http://www.mme.gov.br>. Acesso em: 14 out. 2003.

\section{MINISTÉRIO DE MINAS E}

ENERGIA. Modelo Institucional do Setor Elétrico. Brasília, dez. 2003b, 60 p. Disponível em: <http://www.mme.gov.br> Acesso em: 22 dez. 2003.

\section{MODIANO, E. M.}

Elasticidade-renda e preço da demanda de energia elétrica no Brasil. Rio de Janeiro: Departamento de Economia - PUC/RJ, 1984. (Texto para Discussão, n. 68).

MOREIRA, A. R. B. FIORÊNCIO, A.; LOPES, H. F. Um modelo de previsão do PIB, inflação e meios de pagamento. Rio de Janeiro: IPEA, nov. 1996. (Texto para Discussão, n. 446). 
NARAYAN, P. K.; SMYTH, R. The residential demand for electricity in Australia: na application of the bounds testing approach to cointegration. Energy Policy, p. 1-8, 2003.

NORDIN, J. A. A proposed modification of Taylor's demand analysis: comment. The Bell Journal of Economics, v. 7, p. 719-721, 1976

PIRES, J. C. L. O processo de reformas do setor elétrico brasileiro. Revista do BNDES. Rio de Janeiro, v. 6, n. 12, p. 137-168, dez. 1999

PIRES, J. C. L.;

GOSTKORZEWICZ, J.;

GIAMBIAGI, F. O cenári macroeconômico e as condiçōes de oferta de energia elétrica no Brasil. Rio de Janeiro: BNDES, mar. 2001.

(Texto para Discussão, n. 85).

SCHMIDT, C. A. J.; LIMA, M. A.

A demanda por energia elétrica no Brasil. Revista Brasileira de Economia. Rio de Janeiro, v. 58, p. 67-98, jan./mar. 2004.

SILK, J. I.; JOUTZ, F. L. Short and long-run elasticity's in US residential electricity demand: a co-integration approach. Energy Economics, v. 19, p. 493-513, 1997.

SILVA, S. M. Análise econométrica da demanda de energia elétrica nos setores Residencial-Urbano e Rural do Brasil, 1970/1999. 2001.

Dissertação (Mestrado) -

Universidade Federal de Viçosa,

Viçosa, 2001.
TAYLOR, L. D. The demand for electricity: a survey. Journal of Economics, Arizona, v. 6 p. $74-110,1975$.

VERBEEK, M. A guide to modern econometrics. Nova York: John Wiley \& Sons, Ltd, 2000. 386 p.

\begin{tabular}{l}
$\ldots \ldots \ldots \ldots$ \\
$\vdots$ \\
E-mail de contato dos autores: \\
$:$ leobornacki@yahoo.com.br \\
\hdashline$\ldots \ldots \ldots \ldots$
\end{tabular}

